\title{
A NOTE ON THE HARRIS-SEVAST'YANOV TRANSFORMATION FOR SUPERCRITICAL BRANCHING PROCESSES
}

\author{
H.-J. SCHUH
}

(Received 27 February 1980; revised 18 December 1980)

Communicated by R. L. Tweedie

\begin{abstract}
We show that the Harris-Sevast'yanov transformation for supercritical Galton-Watson processes with positive extinction probability $q$ can be modified in such a way that the extinction probability of the new process takes any value between 0 and $q$. We give a probabilistic interpretation for the new process. This note is closely related to Athreya and Ney (1972), Chapter I.12.

1980 Mathematics subject classification (Amer. Math. Soc.): $60 \mathrm{~J} 80$.

Keywords: supercritical Galton-Watson process, extinction probability, probability generating function, Harris-Sevast'yanov transformation.
\end{abstract}

\section{Introduction}

In this note we shall deal with the Galton-Watson branching process $\left(Z_{n}\right)_{n=0,1,2, \ldots}$. We refer to the books of Harris (1963) and Athreya and Ney (1972) for the basic theory. As usually we assume that $Z_{0} \equiv 1$ and interpret $Z_{n}$ as the number of individuals alive in the $n$th generation. We use the same notation as in Athreya and Ney (1972): $p_{j}=$ probability that an individual has $j$ children, $j=0,1,2, \ldots ; m=\sum_{j=1}^{\infty} j p_{j}$, the offspring mean; $f(s)=\sum_{j=0}^{\infty} p_{j} s^{j}$, $0<s<1$, the probability generating function (p.g.f.) of the offspring distribution (or of $\left.Z_{1}\right) ; f_{n}(s)$ its $n$th iterate (= p.g.f. of $\left.Z_{n}\right) ; q=P\left(Z_{n}=0\right.$ eventually) the extinction probability of $\left(Z_{n}\right)_{n>0}$.

We are interested in the supercritical case, that is $q<1$ (or $1<m<\infty$ ). It is well-known (see Athreya and Ney (1972), Chapter I.10 Theorem 3) that in the

- Copyright Australian Mathematical Society 1982 
case $1<m<\infty$, there always exist positive constants $\left(C_{n}\right)_{n>0}$, such that $Z_{n} / C_{n} \rightarrow W$ a.s. $W$ with $P(W=0)=q$ and $P(0<W<\infty)=1-q$. As $q$ is the only fixed point of $f$ in [0,1[, $q=0$ if and only if $p_{0}=f(0)=0$. Many results in the supercritical case are easily proved for the case $q=0$. Harris (1948) and Sevast'yanov found a transformation which reduces the general case to the case $q=0:$ if $f(0)>0$, consider

$$
\hat{f}(s)=[f((1-q) s+q)-q] /(1-q), \quad 0<s<1,
$$

and the corresponding Galton-Watson process $\left(\hat{Z}_{n}\right)_{n} \cdot \hat{f}(s)$ is a p.g.f. with $\hat{p}_{0}=0$ and $\hat{W}$ has the same distribution as $(1-q) W$ conditioned on the set non-extinction of $\left(Z_{n}\right)_{n}$ (see Harris (1948), Theorem 3.2 and Athreya and Ney (1972), Chapter I.12). It can be shown, for example, (see Athreya and Ney (1972), Chapter I.10 Corollary 4 and Lemma 9) that $\hat{W}$ is absolutely continuous, and thus by the transformation above, $W$ is absolutely continuous on the set of non-extinction.

Athreya and Ney (1972), Chapter I.12, give a probabilistic interpretation of the process $\left(\hat{Z}_{n}\right)_{n}$ (see also Athreya and Karlin (1967), Section 5II). They show that $\hat{Z}_{n}$ can be thought to be the number of individuals of the $n$th generation which have an infinite line of descent.

In this note we shall generalize this transformation such that the extinction probability $\hat{q}$ of $\left(\hat{Z}_{n}\right)_{n}$ can take any value between 0 and $q$ and we shall again interpret $\hat{Z}_{n}$ in a probabilistic way. We further shall give a detailed proof for the branching property of $\left(\hat{Z}_{n}\right)_{n}$, which may be also helpful for the study of Athreya and Ney (1972), Chapter I.12 Theorem 1.

\section{Construction of a process with smaller extinction probability}

Suppose $q>0$ and let $0 \leqslant \hat{q} \leqslant q$, then $z=(q-\hat{q}) /(1-\hat{q}) \in[0, q]$. We proceed in analogy to Athreya and Ney (1972), Chapter 1.12 and construct the graph of the new p.g.f. $\hat{f}(s)$ out of $f(s)$ by "stretching" the square with opposite corners $(z, z)$ and $(1,1)$ in Figure 1 into the unit square, mapping $(z, z)$ into $(0,0)$. The resulting curve will be

$$
\hat{f}(s)=[f((1-z) s+z)-z] /[1-z], \quad 0<s<1 .
$$

As $\hat{f}(0)=(f(z)-z) /(1-z) \geqslant 0$, it is easily checked that $\hat{f}(s)$ is a powerseries with non-negative coefficients $\left(\hat{p}_{j}\right)_{j>0}$ and as $\hat{f}(1)=1, \hat{f}(s)$ is indeed a p.g.f. Furthermore it follows immediately that $\hat{m}=\hat{f}^{\prime}(1)=f^{\prime}(1)=m$, that $\hat{f}_{n}(s)=$ $\left[f_{n}((1-z) s+z)-z\right] /[1-z], 0 \leqslant s \leqslant 1, n=1,2, \ldots$, and that $\hat{f}(\hat{q})=\hat{q}$, that is if $\left(\hat{Z}_{n}\right)_{n}$ has the offspring distribution $\left(\hat{p}_{0}, \hat{p}_{1}, \ldots\right)$, then it dies out with probability $\hat{q}$. If $\hat{q}=0$, then (2) is identical to (1), and if $\hat{q}=q$, then $\hat{f}(s)=f(s)$. 


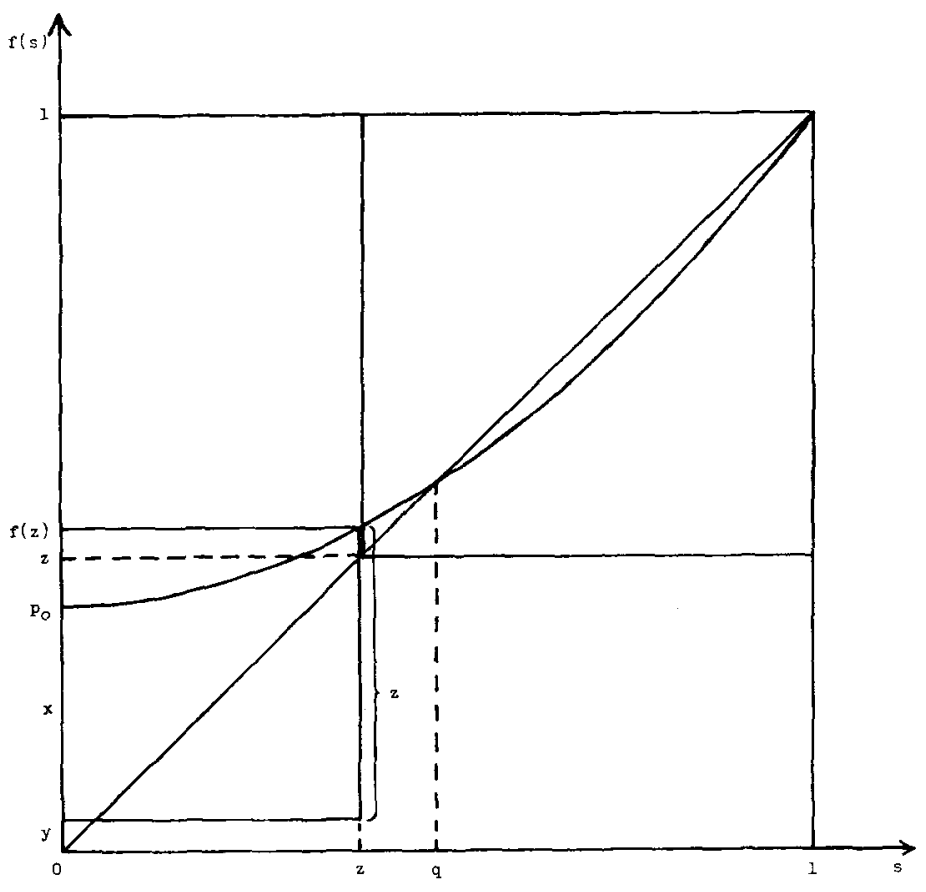

Figure 1

Finally suppose that $1<m<\infty$ and that $\hat{Z}_{n} / \hat{C}_{n} \rightarrow \overrightarrow{\text { a.s. }}$. If $\hat{\phi}(t)$ is the Laplace-transform of $\hat{W}$, then by Athreya and Ney (1972), Chapter I.10 Theorem 3:

$$
\begin{gathered}
\hat{\phi}(t)=\hat{f}(\hat{\phi}(t / m)), \quad \text { or } \\
(1-z) \hat{\phi}(t)+z=f((1-z) \hat{\phi}(t / m)+z) .
\end{gathered}
$$

This implies that for $Z_{n} / C_{n} \rightarrow$ a.s. $W$ there exists a constant $0<c<\infty$ such that $\hat{W}$ conditioned on $\left\{\hat{Z}_{n} \nrightarrow 0\right\}$ and $c \cdot W$ conditioned on $\left\{Z_{n} \nrightarrow 0\right\}$ have the same distribution (see also Harris (1963), Chapter I Theorem 8.2).

\section{Probabilistic interpretation}

In this section we interpret the branching process $\left(Z_{n}\right)_{n}$ as a model for the development of the male part of a population, that is $Z_{n}$ is the number of males in the $n$th generation and $p_{j}, j=0,1, \ldots$, is the probability that a male has $j$ sons. Suppose $q>0$, that is $p_{0}>0$, then decompose $p_{0}=x+y, x, y>0$. We construct now an extended version of the process $\left(Z_{n}\right)_{n}$ : every male in the $n$th 
generation stays either unmarried (and has therefore no children, in particular no sons) with probability $x$, or gets married and has no sons with probability $y$, or gets married and has $j$ sons, $j=1,2, \ldots$, with probability $p_{j}$, independently of all the other males and of the past of the process. All the sons of the males of the $n$th generation form the $(n+1)$ st generation, and we start the process with one male in the 0th generation. Let $Z_{n}^{(1)}$ be the number of males in the $n$th generation and $Z_{n}^{(0)}$ the number of married males (amongst them) which have no sons. Obviously $\left(Z_{n}^{(1)}\right)_{n}$ and $\left(Z_{n}\right)_{n}$ describe the same process, and we will therefore not distinguish them. We define $\left(Z_{n}^{\prime}\right)_{n}=\left(\left(Z_{n}, Z_{n}^{(0)}\right)\right)_{n}$ as the (extended) Galton-Watson process with offspring distribution $p^{\prime}=\left(x, y, p_{1}, p_{2}, \ldots\right)$.

Let $(\Omega, \mathscr{F}, P)$ be a probability space which is large enough to accomodate the process $\left(Z_{n}^{\prime}\right)_{n}$. (The construction is obvious and can be left to the reader).

Definition. A male (alive in any generation) is called a $B$-male, if the (extended) Galton-Watson process formed by his male-descendants dies out and all male-descendants without sons are unmarried, that is each line of descent ends with a bachelor. Otherwise he is called an $A$-male, that is the process of his male-descendants either never dies out (that is infinite line of descent), or at least one of his male-descendants is married but has no sons.

Let $I$ be the male of the 0 th generation, and $n_{0}(\omega)=\max \left\{n \mid Z_{n}(\omega)>0\right\}$, $\omega \in \Omega$, that is $n_{0}=\infty$ on $\left\{Z_{n} \rightarrow \infty\right\}$. We define

$$
\begin{aligned}
& A=\{I \text { is an } A \text {-male }\}=\left\{n_{0}=\infty \text { or } Z_{n}^{(0)}>0 \text { for some } n\right\} ; \\
& B=\{I \text { is a } B \text {-male }\}=\left\{n_{0}<\infty \text { and } Z_{n}^{(0)}=0 \text { for all } n\right\} ; \\
& A \cup B=\Omega .
\end{aligned}
$$

Let $z=P(B)$, then $P(A)=1-z$ and $z<q$. For $j \geqslant 1: P\left(B \cap\left\{Z_{1}=j\right\}\right)=$ $P\left(B \mid Z_{1}=j\right) p_{j}=z^{j} p_{j}$, and for $j=0: P\left(B \cap\left\{Z_{1}=0\right\}\right)=P(I$ stays unmarried $)$ $=x$. Hence $z=P(B)=\sum_{j=0}^{\infty} P\left(B \cap\left\{Z_{1}=j\right\}\right)=x+\sum_{j=1}^{\infty} p_{j} z^{j}$, or

$$
x=p_{0}-f(z)+z \text { and } y=p_{0}-x=f(z)-z .
$$

Remark. That $p_{0} \geqslant f(z)-z$ follows also from $z \geqslant z \sum_{j=1}^{\infty} p_{j}>\sum_{j=1}^{\infty} p_{j} z^{j}=$ $f(z)-p_{0}$.

We define $\hat{Z}_{n}$ as the number of $A$-males amongst the $Z_{n}$ males of the $n$th generation. Obviously $\hat{Z}_{n} \equiv 0$ on $B$. 
THEOREM 1. Conditioned on $A,\left(\hat{Z}_{n}\right)_{n=0,1} \ldots$ is a Galton-Watson process whose offspring distribution has the p.g.f. $\hat{f}(s)$, defined in (2).

REMARK. By (3), $x$ and $y$ can graphically be found as indicated in Figure 1 .

Before we prove the theorem we need the following lemma which can be checked easily.

LEMMA. Suppose $E_{1}, \ldots, E_{n}$ are mutually exclusive events and for another event $D, P\left(D \mid E_{1}\right)=P\left(D \mid E_{2}\right)=\ldots=P\left(D \mid E_{n}\right)=p_{D}$, then also

$$
P\left(D \mid E_{1} \cup \cdots \cup E_{n}\right)=p_{D}
$$

Proof of Theorem 1. Obviously $\hat{z}_{0} \equiv 1$ on $A$.

Step 1. We shall show that, conditioned on $A \cap\left\{\hat{Z}_{n}=j\right\}, \hat{Z}_{n+1}$ is distributed like the sum of $j$ i.i.d. random variables whose distribution does not depend on $n$, that is $\left(\hat{Z}_{n}\right)_{n}$ is a Galton-Watson process.

On $\left\{Z_{n}=k\right\}, k \geqslant 0$, let $I_{1}, \ldots, I_{k}$ be the $k$ males of the $n$th generation and $M_{i}$ the number of sons of $I_{i}$, which are $A$-males $\left(M_{i}=0,1,2, \ldots\right)$. Let further

$$
\eta_{i}= \begin{cases}1 & \text { if } I_{i} \text { is married and has no sons, } \\ 0 & \text { otherwise, }\end{cases}
$$

$i=1, \ldots, k$, (that is $\eta_{i}=1 \Rightarrow M_{i}=0$ ). $I_{i}$ is an $A$-male if and only if $M_{i}>0$ or $\eta_{i}=1$. The branching property of $\left(Z_{n}^{\prime}\right)_{n}$ implies that

$$
\begin{gathered}
\left(\left(M_{i}, \eta_{i}\right)\right)_{1<i<k} \text { are i.i.d., do not depend on } n \text {, and } \\
\hat{Z}_{n+1}=\sum_{i=1}^{k} M_{i} \text { on }\left\{Z_{n}=k\right\} .
\end{gathered}
$$

Let $1 \leqslant j \leqslant k$. For $l \geqslant 0$ and $1 \leqslant i_{1}<i_{2}<\cdots<i_{j} \leqslant k$, let $1 \leqslant i_{j+1}$ $<\cdots<i_{k} \leqslant k$ be such that $\left\{i_{1}, \ldots, i_{k}\right\}=\{1, \ldots, k\}$ and define

$$
\begin{aligned}
D & =\left\{\hat{Z}_{n+1}=l ; Z_{n}=k\right\}=\left\{\sum_{i=1}^{k} M_{i}=l\right\} \\
E_{i_{1}, \ldots, i_{j}} & =\left\{Z_{n}=k, I_{i_{1}}, \ldots, I_{i_{j}} \text { are } A \text {-males, } I_{i_{j+1}}, \ldots, I_{i_{k}} \text { are } B \text {-males }\right\} \\
& =\bigcap_{r=1}^{j}\left\{M_{i_{r}}>0 \text { or } \eta_{i_{r}}=1\right\} \cap \bigcap_{r=j+1}^{k}\left\{M_{i_{r}}=0 \text { and } \eta_{i_{r}}=0\right\}
\end{aligned}
$$


The $E_{i_{1}, \ldots, i}$ 's are mutually exclusive and by (5), $P\left(D \mid E_{i_{1}, \ldots, j}\right)=p_{D}$ independent of $i_{1}, \ldots, i_{j}$. Hence, employing (4) and (5),

$$
\begin{aligned}
& P\left(\hat{Z}_{n+1}=l \mid \hat{Z}_{n}=j, Z_{n}=k\right) \\
& =P\left(\sum_{i=1}^{k} M_{i}=l \mid Z_{n}=k \text { and } j \text { of these males are } A \text {-males }\right) \\
& =P\left(\sum_{i=1}^{k} M_{i}=l \mid \bigcup_{\left\{i_{1}, \ldots, i_{j}\right\} \subset\{1, \ldots, k\}} E_{i_{1}, \ldots, i j}\right)=P\left(\sum_{i=1}^{k} M_{i}=l \mid E_{1, \ldots, j}\right) \\
& =P\left(\sum_{i=1}^{j} M_{i}=l \mid E_{1, \ldots, j}\right)=P\left(\sum_{i=1}^{j} M_{i}=l \mid M_{r}>0 \text { or } \eta_{r}=1 \text { for } 1<r<j\right) \\
& =P\left(\sum_{i=1}^{j} N_{j}=l\right), \quad \text { where } N_{1}, \ldots, N_{j} \text { are i.i.d. with distribution } \\
& \quad P\left(N_{i}=r\right)=P\left(M_{i}=r \mid M_{i}>0 \text { or } \eta_{i}=1\right), \quad r=0,1, \ldots
\end{aligned}
$$

As for $j \geqslant 1,\left\{\hat{Z}_{n}=j\right\} \subset A$,

$$
\begin{aligned}
P\left(\hat{Z}_{n+1}\right. & \left.=l \mid\left(\hat{Z}_{n}=j\right) \cap A\right)=P\left(\hat{Z}_{n+1}=l \mid \hat{Z}_{n}=j\right) \\
& =\sum_{k=j}^{\infty} P\left(\hat{Z}_{n+1}=l \mid \hat{Z}_{n}=j, Z_{n}=k\right) P\left(Z_{n}=k \mid \hat{Z}_{n}=j\right) \\
& =P\left(\sum_{i=1}^{j} N_{i}=l\right) \sum_{k=j}^{\infty} P\left(Z_{n}=k \mid \hat{Z}_{n}=j\right)=P\left(\sum_{i=1}^{j} N_{i}=l\right) .
\end{aligned}
$$

On $\left\{\hat{Z}_{n}=0\right\} \cap A, Z_{n}$ consists only of $B$-males and hence $Z_{n+1}$ consists only of $B$-males, that is

$$
P\left(\hat{Z}_{n+1}=0 \mid\left(\hat{Z}_{n}=0\right) \cap A\right)=1=P\left(\sum_{i=1}^{0} N_{i}=0\right)
$$

Hence on $A, \hat{Z}_{n+1}$ is distributed like $\sum_{i=1}^{\hat{Z}_{n}} N_{i}$, that is $\left(\hat{Z}_{n}\right)_{n}$ is a Galton-Watson process.

Step 2. It is left to show that $\left(\hat{Z}_{n}\right)_{n}$ conditioned on $A$ has the offspring distribution which corresponds to $\hat{f}(s)$. It is enough to calculate the p.g.f. of $\hat{Z}_{1}$ conditioned on $A$.

$$
\begin{aligned}
P(A) & =1-z ; P\left(\left(\hat{Z}_{1}=0\right) \cap A\right)=P(I \text { is married but has no sons }) \\
& =y=f(z)-z \text { by }(3) .
\end{aligned}
$$


For $1 \leqslant j \leqslant k: P\left(\hat{Z}_{1}=j \mid Z_{1}=k\right)=P(j$ of the $k$ males are $A$-males $)=$ $\left(\begin{array}{l}k \\ j\end{array}\right)(1-z)^{\dot{z}} z^{k-j}$ by the branching property of $\left(Z_{n}^{\prime}\right)_{n}$. As $\left(\hat{Z}_{1}=j\right) \subset A$,

$$
\begin{aligned}
P\left(\left(\hat{Z}_{1}=j\right) \cap A\right) & =P\left(\hat{Z}_{1}=j\right)=\sum_{k=j}^{\infty} P\left(\hat{Z}_{1}=j \mid Z_{1}=k\right) P\left(Z_{1}=k\right) \\
& =\sum_{k=j}^{\infty}\left(\begin{array}{l}
k \\
j
\end{array}\right)(1-z)^{j} z^{k-j} p_{k} .
\end{aligned}
$$

Hence

$$
\begin{aligned}
\sum_{j=0}^{\infty} P\left(\hat{Z}_{1}=j \mid A\right) s^{j} & =(1-z)^{-1}\left(f(z)-z+\sum_{j=1}^{\infty} \sum_{k=j}^{\infty}\left(\begin{array}{l}
k \\
j
\end{array}\right)(1-z)^{j} s^{j} z^{k-j} p_{k}\right) \\
& =(1-z)^{-1}\left(f(z)-z+\sum_{k=1}^{\infty} p_{k} \sum_{j=1}^{k}\left(\begin{array}{l}
k \\
j
\end{array}\right)(1-z)^{j} s^{j} z^{k-j}\right) \\
& =(1-z)^{-1}\left(f(z)-z+\sum_{k=1}^{\infty} p_{k}\left(((1-z) s+z)^{k}-z^{k}\right)\right) \\
& =(1-z)^{-1}(f(z)-z+f((1-z) s+z)-f(z))=\hat{f}(s) .
\end{aligned}
$$

REMARK. In the case $\hat{q}=0$ we have the following simplifications: $z=q$, $x=p_{0}, y=0:$ an $A$-male is a male with an infinite line of descent, and $\eta_{i} \equiv 0$.

The following two results can be shown in a similar way as the Theorems 2 and 3 of Athreya and Ney (1972), Chapter I.12.

Theorem 2. On $\left\{Z_{n} \rightarrow \infty\right\}$,

$$
\hat{Z}_{n} / Z_{n} \rightarrow(1-z) \text {. }
$$

If $\hat{Z}_{n}=0$ for some $n$, then also $Z_{n^{\prime}}=0$ for some $n^{\prime}$.

THEOREM 3. Conditioned on $B$, the process $\left(Z_{n}\right)_{n}$ is a subcritical Galton-Watsan process (that is $E\left(Z_{1} \mid B\right)<1$ ) whose offspring distribution has the p.g.f.

$$
\begin{aligned}
& \quad \tilde{f}(s)=[f(z \cdot s)-(f(z)-z)] / z, \quad 0<s<1 . \\
&\left(\Leftrightarrow \tilde{m}=\tilde{f^{\prime}}(1)=f^{\prime}(z)<1 .\right)
\end{aligned}
$$

ReMARK. (a) On $B, Z_{n}$ is the number of $B$-males in the $n$th generation.

(b) The graph of $\tilde{f}(s)$ can be constructed out of the graph of $f(s)$ by "stretching" the square with opposite corners $(0, y)$ and $(z, f(z))$ in Figure 1 into the unit square, mapping $(0, y)$ into $(0,0)$ and $(z, f(z))$ into $(1,1)$. 


\section{References}

K. B. Athreya and S. Karlin (1967), 'Limit theorems for the split times of branching processes', $J$. Math. Mech. 17, 257-277.

K. B. Athreya and P. E. Ney (1972), Branching Processes (Springer-Verlag, Berlin).

T. E. Harris (1948), 'Branching processes', Ann. Math. Statist. 19, 474-494.

T. E. Harris (1963), The Theory of Branching Processes (Springer-Verlag, Berlin).

Department of Statistics

University of Melbourne

Melbourne, Victoria

Australia 